\title{
Relación entre los motivos para la práctica físico-deportiva y las experiencias de flujo en jóvenes: diferencias en función del sexo*
}

\author{
Relationship between Motives for Exercise and Sports Practice \\ and Flow Experiences in Youth: Gender Differences
}

Recibido: agosto 11 de 2010 | Revisado: abril 20 de 2011 | Aceptado: noviembre 11 de 2011

\author{
MANUEL G. JiMÉNEZ-TORRES ** \\ DÉBORA GODOY-IZQUIERDO \\ JUAN F. GODOY GARCÍA \\ Universidad de Granada, España
}

SICI: 1657-9267(201209)11:3<909:RMPEJS>2.0.TX;2-R

Para citar este artículo: Jiménez-Torres, M. G., Godoy-Izquierdo, D. \& Godoy, G. J. F. (2012). Relación entre los motivos para la práctica físico-deportiva y las experiencias de flujo en jóvenes: diferencias en función del sexo. Universitas Psychologica, 11(3), 909-920.

Trabajo de investigación.

** Departamento de Personalidad, Evaluación y Tratamiento Psicológico. Facultad de Psicología, Campus de Cartuja, s/n. 18071, Granada, España. Correoselectrónicos: mjitor@ugr.es, deborag@ugr. es, jgodoy@ugr.es

\section{RES U MEN}

El presente trabajo estudia la relación entre práctica físico-deportiva, motivos para realizarla y flujo experimentado en dicha práctica en una muestra de 101 jóvenes españoles. Los participantes respondieron autoinformes de actividad físico-deportiva, motivos para realizar esa actividad (Capdevila, 2003) y experiencias de flow en la misma (Jackson \& Eklund, 2002). Los resultados indicaron que los hombres realizan más ejercicio y deporte que las mujeres, manifiestan una mayor motivación intrínseca y experimentan un mayor nivel de flujo en la actividad físico-deportiva. El flujo experimentado y los motivos intrínsecos se mostraron como factores predictores de la práctica físico-deportiva, mientras que los motivos extrínsecos no predijeron dicha práctica. Los resultados anteriores sugieren que fomentar motivos de diversión y experiencias subjetivas positivas como el flujo ayudarán a incrementar la adherencia a la práctica físico-deportiva en jóvenes.

Palabras clave autores:

Ejercicio físico, flujo, motivos, motivación.

Palabras clave descriptores:

Psicología deportiva, deporte, actividad física.

\section{A B S T R A C T}

In this study we explored the relationship between exercise-sports practice, motives for practice and flow experienced during such activities in a sample of Spanish young adults. 101 participants responded self-reports on exercisesports practice, motives (Capdevila, 2003) and states of flow (Jackson \& Eklund, 2002). The results indicated that, compared to women, physical practice was higher among men, who also showed a higher intrinsic motivation and experienced flow at a higher degree. Flow and intrinsic motives predicted physical practice, whilst extrinsic motives could not predict it. These results point out that promoting enjoyment-related motives and positive subjective experiences such as flow might help in enhancing adherence to exercise and sports among young people.

Key words authors:

Exercise, Flow, Motives, Motivation.

Key words plus:

Sport Psychology, Sport, Physical Activity. 


\section{Introducción}

La juventud es una etapa decisiva en la adquisición y consolidación de estilos de vida saludables y la práctica de ejercicio físico y deporte se considera un pilar fundamental para la futura salud del individuo. Numerosos estudios destacan el sedentarismo entre las principales causas de preocupación para la salud pública y subrayan la necesidad de conocer los diferentes factores que explican su incidencia y prevalencia (Biddle \& Mutrie, 2008; Dishman, 1988, 1991).

Los estudios descriptivos y epidemiológicos acerca de la actividad físico-deportiva realizada por los jóvenes concluyen generalmente que es insuficiente, y que se va reduciendo conforme se sale de la adolescencia y primera juventud. Por otro lado, casi todas las investigaciones concuerdan en que las mujeres realizan menos actividad física que los hombres (Weinberg et al., 2000). Piéron (2002), al estudiar la frecuencia de participación en actividades físicas y deportivas en función del sexo y la edad en una muestra de jóvenes españoles de entre 14 y 18 años, encontró que la proporción de jóvenes que realizan una actividad deportiva con una frecuencia lo bastante elevada como para proporcionar efectos beneficiosos era de un 30-50\% en los hombres y en torno a un $20 \%$ en las mujeres. Chillón, Tercedor, Delgado y González-Gross (2002) también obtienen diferencias entre hombres y mujeres españoles en cuanto al porcentaje de individuos que realizan actividad física, siendo este menor en el caso de la mujeres. Así pues, parece evidente un menor nivel de práctica y adherencia entre las mujeres y esto se refleja a lo largo de todas las edades estudiadas, incluyendo la edad escolar (Tercedor \& Delgado, 1998) y la primera adultez (Jiménez, Martínez, Miró \& Sánchez, 2008; Sánchez-Barrera, Pérez \& Godoy, 1995; Varo et al., 2003). Otras investigaciones confirman que tanto los hombres como las mujeres disminuyen la práctica deportiva a medida que aumenta la edad, y que esta disminución es más acusada en las mujeres (Pastor, Balaguer \& GarcíaMerita, 1999).

Entre los factores psicológicos y psicosociales que se han estudiado para explicar la práctica de actividad física y deporte se encuentran los de tipo motivacional. La motivación se refiere a los aspectos de intención y activación en relación con una conducta en términos de energía o intensidad, dirección, persistencia y finalidad (Ryan \& Deci, 2000). Entre los principales motivos que explican la participación de los jóvenes en la actividad física están los de diversión, aprender nuevas habilidades, mejorar la forma física, competir, deseo de logro y rendimiento, estar y relacionarse con otros iguales, mejora de salud u obtener éxito. No obstante, parecen existir diferencias entre hombres y mujeres en los diferentes motivos que les llevan a practicar actividades físico-deportivas. Así, las mujeres suelen perseguir más el control de peso y la mejora del aspecto externo así como la relación social, mientras que los hombres están más inclinados hacia el logro de una mejora en la forma física, el rendimiento y la competición (Cruz, 2001; Weinberg et al., 2000). Además, las investigaciones sobre el tema también destacan otros motivos que hacen referencia al placer intrínseco que conlleva realizar la actividad físico-deportiva (Dishman, Sallis \& Orensein, 1985). Así, la alegría o el placer se presentan a menudo como el motivo más importante para realizar y, sobre todo, para mantener la práctica.

Una teoría motivacional que está ganando cada vez mayor peso en el ámbito de las conductas saludables es la Teoría de la Autodeterminación (Self-Determination Theory-SDT) de Deci y Ryan $(1985,2002)$ y Ryan y Deci $(2000)$. Esta teoría también ha sido aplicada a la conducta de ejercicio físico de tipo recreativo o asociada al tiempo libre y ocio con jóvenes (Daley \& Duda, 2006; Sicilia, Águila, Muyor, Orta \& Moreno, 2009; Wilson, Rodgers, Fraser \& Murray, 2004). La teoría de la autodeterminación pretende dar cuenta de por qué la gente inicia, persiste o cesa su implicación en la práctica de ejercicio físico (Hagger \& Chatzisarantis, 2007). Conocer los procesos motivacionales en el caso de la conducta de ejercicio físico nos ayudaría a optimizar el inicio y la continuación de la práctica de actividad física, así como a reducir el abandono. En esta teoría se distinguen distintos estados u orientaciones motivacionales dependiendo del grado de internalización e integración de la 
autodeterminación de la actividad, es decir, del grado en que la regulación de la conducta emana de la propia persona (Deci \& Ryan, 1985; Ryan \& Deci, 2000). En la SDT se proponen seis estados motivacionales en un continuo que va desde el estado de amotivación al de motivación intrínseca (la más autodeterminada), pasando por cuatro estados de motivación extrínsecamente determinada (de más extrínsecamente orientada a más intrínsecamente orientada: orientación externa, orientación introyectada, orientación identificada y orientación integrada).

En el caso de la motivación intrínseca, la conducta se realiza por la diversión que supone, por el simple placer interno de hacerla o por las satisfacciones inherentes a la actividad, en ausencia de refuerzos, presiones, coerción o influencias externas. Con este tipo de orientación, el disfrute, desarrollo personal y autorrealización son mayores y el compromiso y la adherencia a largo plazo por la actividad serán máximos (Ryan \& Deci, 2000). Las orientaciones motivacionales más autodeterminadas se asocian a su vez a estados emocionales más positivos, incluyendo estados de flow (Csikszentmihalyi, 1975, 1997; Deci \& Ryan, 1985).

En los últimos años ha irrumpido con fuerza un movimiento especialmente interesado en estudiar los aspectos positivos y las fortalezas de las personas, y uno de los fenómenos investigados con más rigor en el campo de la Psicología Positiva es el de la experiencia óptima oflow (Seligman \& Csikszentmihalyi, 2000). En sus orígenes, el término flow o flujo fue utilizado por Csikszentmihalyi (1975) para referirse a un estado mental óptimo en el que un individuo siente eficiencia cognitiva, está profundamente involucrado en la actividad que realiza, altamente motivado y al mismo tiempo experimenta un alto nivel de gozo.

Diversos estudios han demostrado que la práctica de una actividad físico-deportiva está asociada a experiencias profundas y frecuentes de flujo (Csikszentmihalyi, 1996). Csikszentmihalyi y Csikszentmihalyi (1998), al estudiar mediante el Método de Muestreo de Experiencias (ESM) la calidad de las experiencias subjetivas de adolescentes en distintos ámbitos de su vida, demostraron que la actividad físico-deportiva en particular es una de las actividades en las que con mayor frecuencia se experimenta flujo, y que los adolescentes son mucho más felices cuando hacen deporte o realizan un tipo de ocio activo que cuando participan en otras actividades de ocio pasivo, como ver la televisión o pasear por unos grandes almacenes.

El estado de flujo ha sido introducido como un elemento que contribuye a explicar la práctica de la actividad físico-deportiva en relación con el ocio y el tiempo libre entre practicantes españoles (García-Calvo, Jiménez, Santos-Rosa, Reina \& Cervelló, 2008; Godoy-Izquierdo, Molina \& Godoy, en prensa; Sicilia et al., 2009).

En el ámbito del deporte ha sido descrito con base en nueve características (Jackson \& Csikszentmihalyi, 2002): 1) Equilibrio entre reto y habilidad. Cuando una persona debe enfrentarse a una actividad deportiva, los retos que se proponga en relación con la misma deben estar en equilibrio con las habilidades que posea para llevarla a cabo para evitar estados de ansiedad o de aburrimiento. 2) Fusión entre la atención y la acción. Se refiere al hecho de lograr la unión entre la mente y el cuerpo para realizar una actividad de forma más fluida. Se evita así la pérdida de tiempo que supone detenerse a pensar aquello que se ha de llevar a cabo. 3) Metas claras. Es importante que la persona que realiza una actividad deportiva tenga claro cuáles son sus objetivos, las metas que persigue en la ejecución de la misma. 4) Feedback sin ambigüedad. Está relacionado con la anterior en cuanto que si la persona tiene claros cuáles son sus objetivos es más probable que sepa si la actividad llevada a cabo la está realizando de modo correcto para lograr los objetivos propuestos (p. e., feedback cinestésico). 5) Concentración en la tarea. Se trata de centrarse en la tarea que se está realizando y no prestar atención a estímulos ajenos a la propia tarea que actúen como distractores (p.e., pensamientos negativos). 6) Sensación de control. Cuando la persona siente que tiene control sobre la tarea que está realizando es más probable que se encuentre en un estado psicológico óptimo. La sensación de control será mayor cuando haya un equilibrio retos-habilidades y también en la medida en que se tengan los obje- 
tivos claros y se presente un feedback inequívoco. 7) Pérdida de conciencia del propio ser. Se refiere al hecho de mantenerse alejado de preocupaciones y temores sobre la actividad que se deba llevar a cabo, lo que se logra cuando están presentes la unión pensamiento-acción y la concentración en la tarea. 8) Transformación del tiempo. La persona puede percibir el paso del tiempo de modo alterado, bien alargado (los segundos pueden parecer horas) o bien reducido (el tiempo pasa volando). Esta flexibilidad de percepción permite que la mente pueda adaptarse según los requerimientos de la situación. 9) Experiencia autotélica. Está relacionada con el disfrute que experimenta una persona al realizar una tarea, de modo que realizarla se convierte en un objetivo en sí mismo por el mero placer que reporta realizarla, sin buscar otro tipo de recompensa externa (p. e., resultados, premios). La experiencia autotélica a partir de la práctica físico-deportiva favorece la adherencia a la misma por el disfrute y satisfacción que experimenta la persona al realizarla (Csikszentmihalyi, 1996).

Consideramos que el estudio del flujo, así como de los motivos asociados a dicha práctica, puede ser relevante para comprender por qué algunos jóvenes mantienen una alta adherencia a la práctica físicodeportiva, mientras que otros no practican en absoluto, o bien la abandonan al poco tiempo de iniciarla.

Teniendo en cuenta lo anterior, el presente trabajo se ha centrado en estudiar las relaciones entre el volumen de práctica físico-deportiva (frecuencia y duración semanales), los motivos para llevar a cabo la práctica físico-deportiva y el flujo experimentado en la misma, considerando las diferencias debidas al sexo de los participantes. Concretamente, se quiso establecer las diferencias entre hombres y mujeres en cuanto a la práctica de actividad misma, así como comprobar si existen diferencias entre hombres y mujeres en cuanto a los motivos para la realización de actividad físicodeportiva y al flujo experimentado con esa actividad. También se quiso averiguar las relaciones entre práctica físico-deportiva, los motivos para su realización y el flujo experimentado, tanto en hombres como en mujeres, y comprobar si la práctica físico-deportiva podía predecirse a par- tir del flujo experimentado y del tipo de motivos (intrínsecos vs. extrínsecos), tanto en hombres como en mujeres.

\section{Método}

\section{Participantes}

Participaron voluntariamente en el estudio 101 jóvenes (54 hombres y 47 mujeres) con edades comprendidas entre los 16 y 20 años $(M=16.75$; $D E=0.78)$. Los participantes estaban cursando estudios de Bachillerato en tres Institutos de Enseñanza Secundaria públicos de Granada (España). Los datos de otros 65 jóvenes que no practicaban ninguna actividad físico-deportiva no fueron tenidos en cuenta en los análisis.

\section{Instrumentos}

Para la recolección de datos se utilizaron las siguientes medidas:

1.- Ficha de datos generales: se registraron datos sobre sexo, edad y curso académico.

2.- Autoinforme sobre la práctica de actividad físico-deportiva extraescolar, incluyendo número de días semanales de práctica y tiempo dedicado a las sesiones durante la semana inmediatamente anterior a la medida. Los parámetros considerados en los análisis fueron los de frecuencia y duración de la práctica.

3.- La experimentación de estados de flujo durante la práctica de ejercicio físico o deporte fue evaluada a través de un instrumento elaborado expresamente para este estudio y que incluye los 9 componentes descritos por Csikszentmihalyi y Csikszentmihalyi (1998): equilibrio entre retos y habilidades, fusión acción-atención, sensación de control, feedback sin ambigüedad, concentración total, metas claras, pérdida de conciencia del ser, alteración de la percepción del paso del tiempo y experiencia autotélica. Cada dimensión fue medida a través de un ítem con una escala de respuesta tipo Likert de 1 (total desacuerdo) a 7 (total acuerdo) puntos. Por ejemplo, para medir la experiencia au- 
totélica se utilizó el ítem "Me gusta tanto realizar ejercicio físico o deporte que estoy deseando que llegue la próxima sesión para volver a vivir esa experiencia". La adecuación de los 9 ítems utilizados para medir el flujo fue contrastada mediante un estudio piloto, obteniendo una fiabilidad test-retest de entre 0.77 y 0.86 para 8 de las 9 dimensiones para un intervalo de 2 semanas. Solo la dimensión referida a la pérdida de conciencia del ser obtuvo una fiabilidad menor (0.5), aunque se optó por mantenerla dado que dicho valor puede considerarse aceptable para fiabilidad test-retest (Morales, 2007). El coeficiente Alfa de Cronbach obtenido en este estudio para el instrumento completo fue de 0.79 .

4.- Los motivos para la realización de una actividad físico-deportiva extraescolar fueron evaluados con base en la clasificación que se establece en el Autoinforme sobre Motivos para la Práctica del Ejercicio Físico (Exercise Motivations Inventory [EMI-2], Markland \& Hardy, 1993, adaptación de Capdevila, 2003): motivos psicológicos (control del estrés, vigor, desafío), motivos interpersonales (reconocimiento social, afiliación, competencia), motivos de salud (urgencias de salud, prevención, salud positiva), motivos corporales (control del peso, imagen corporal) y motivos de forma física (fuerza, resistencia, agilidad, flexibilidad). Además, se ha incluido una categoría adicional referente al motivo diversión. El instrumento incluye en total 8 ítems, todos ellos con una escala de respuesta tipo Likert de 1 (total desacuerdo) a 7 (total acuerdo) puntos. Dos de los ítems se refieren a motivos intrínsecos (me divierte hacerlo, me gusta competir). Los 6 ítems restantes estaban relacionados con motivos extrínsecos: a) psicológicos (me sube el ánimo, me reduce la ansiedad), b) físicos (para mejorar mi salud, para controlar el peso e imagen, para mejorar mi forma física) y c) sociales (para relacionarme con otros). Siguiendo la teoría de Deci y Ryan (1985), el instrumento anterior permite identificar a las personas que están motivadas intrínsecamente o extrínsecamente. Las primeras practicarían ejercicio para obtener satisfacción y disfrute por la mera participación (por ejemplo, diversión o desafío), mientras que las segundas lo practicarían para obtener recompensas externas a la propia conducta (por ejemplo, perder peso, mejorar el aspecto corporal u obtener reconocimiento social). La fiabilidad temporal (mediante test-retest) del instrumento fue superior a 0.8 (Capdevila, Niñerola \& Pintanel, 2004).

\section{Procedimiento}

El protocolo de evaluación fue administrado a los participantes por uno de los investigadores durante una jornada lectiva tras haber obtenido permiso previamente tanto de la dirección del centro como del profesor correspondiente. Se solicitó a los participantes su colaboración de manera anónima y voluntaria en un estudio que pretendía examinar la dedicación del tiempo semanal a distintas actividades físico-deportivas extraescolares, así como la experiencia proporcionada por cada una de ellas. Se insistió especialmente en la sinceridad de las respuestas. Los participantes no recibieron ninguna compensación económica ni académica y ninguno rehusó a la contestación de los cuestionarios. Todos los participantes que contestaron que no realizaban ninguna actividad físico-deportiva extraescolar fueron excluidos de los análisis de este estudio.

\section{Diseño de estudio y análisis de datos}

Se utilizó un diseño no experimental transversal de tipo descriptivo correlacional. El tratamiento estadístico de los datos se ha realizado con el programa SPSS 16.0 (Stadistical Package for Social Science, 2008). Con el fin de comprobar la exactitud de los datos introducidos y conocer las características de los mismos, se realizaron análisis preliminares y exploratorios. Dado que, aunque el supuesto paramétrico de normalidad no se cumplía para las variables de estudio (prueba Kolmogorov-Smirnov $p<0.05$ ), la prueba de Levene confirmó la homogeneidad de las varianzas $(p>0.05)$, se decidió utilizar pruebas paramétricas en los análisis estadísticos. El nivel de significación para todos los análisis se fijó en $p<0.05$ (bilateral). Se llevaron a cabo análisis descriptivos, comparaciones de muestras independientes mediante la prueba $t$ de Student, correlaciones bivariadas de Pearson y análisis de regresión lineal múltiple por pasos sucesivos. 


\section{Resultados}

Diferencias entre hombres y mujeres en cuanto a frecuencia, duración de la sesión y minutos semanales de realización de actividad físico-deportiva extraescolar

Como se muestra en la Tabla 1, no existen diferencias significativas entre hombres y mujeres en cuanto a la frecuencia semanal de realización de ejercicio físico, pero sí en cuanto al tiempo que dura la sesión y también en cuanto a los minutos semanales dedicados a la práctica físico-deportiva extraescolar. Los hombres tienden a hacer sesiones más duraderas de actividad físico-deportiva y, por tanto, el tiempo de práctica semanal es notablemente mayor en su caso que en el de las mujeres.
Diferencias entre hombres y mujeres en cuanto a los motivos de realización de actividad físico-deportiva extraescolar y en cuanto al flujo experimentado con esa actividad

Las diferencias entre hombres y mujeres en cuanto a los motivos y en cuanto a las experiencias de flujo se muestran también en la Tabla 1. En los hombres tienen un peso significativamente mayor que en las mujeres los motivos de diversión y gusto por competir. Por el contrario, no se encontraron diferencias significativas para el resto de tipo de motivos. Cuando se categorizó los motivos en cuatro dimensiones, efectos psicológicos, físicos, sociales y de divertimento, se encontraron diferencias significativas sólo para la dimensión de divertimento. Cuando se cate-

\section{TABLA 1}

Comparaciones entre hombres y mujeres en parámetros de práctica físico-deportiva, motivos para la práctica y flujo experimentado

\begin{tabular}{|c|c|c|c|}
\hline \multirow[b]{2}{*}{ Variables } & \multicolumn{2}{|c|}{$M(D E)$} & \multirow[b]{2}{*}{$t$} \\
\hline & $\begin{array}{l}\text { Hombres } \\
(N=54)\end{array}$ & $\begin{array}{l}\text { Mujeres } \\
(N=47)\end{array}$ & \\
\hline $\begin{array}{l}\text { Parámetros de realización de ejercicio físico } \\
\text { Frecuencia semanal (no de días) } \\
\text { Duración de la sesión (minutos) } \\
\text { Minutos semanales }\end{array}$ & $\begin{array}{c}4(1.76) \\
90.93(35.57) \\
363.89(212.69)\end{array}$ & $\begin{array}{c}3.77(1.75) \\
75.74(41.2) \\
277,66(171.48)\end{array}$ & $\begin{array}{l}\text { n.s. } \\
1.993 * \\
2.252 *\end{array}$ \\
\hline $\begin{array}{l}\text { Motivos } 1 \\
\text { 1. Para subir el ánimo } \\
\text { 2. Para reducir la ansiedad } \\
\text { 3. Para mejorar la salud } \\
\text { 4. Para reducir peso y mejorar imagen } \\
\text { 5. Para mejorar forma física } \\
\text { 6. Para relacionarse } \\
\text { 7. Para divertirse } \\
\text { 8. Por el gusto de competir }\end{array}$ & $\begin{array}{c}5.69(1.27) \\
4.56(1.83) \\
6.17(1.09) \\
5(1.9) \\
6.24(1.26) \\
5.2(1.39) \\
6.63(0.71) \\
5.24(1.88) \\
\end{array}$ & $\begin{array}{c}5.79(0.98) \\
5(1.59) \\
6.23(1.01) \\
5.34(1.7) \\
6.09(1.12) \\
5.26(1.58) \\
6.19(0.92) \\
4.17(2)\end{array}$ & $\begin{array}{l}\text { n.s. } \\
\text { n.s. } \\
\text { n.s. } \\
\text { n.s. } \\
\text { n.s. } \\
\text { n.s. } \\
2.691 * * \\
2.772 * * \\
\end{array}$ \\
\hline $\begin{array}{l}\text { Motivos } 2 \\
\text { 9. Por los efectos psicológicos }(1+2) \\
\text { 10. Por los efectos físicos }(3+4+5) \\
\text { 11. Por los efectos sociales }(6) \\
\text { 12. Por divertimento }(7+8)\end{array}$ & $\begin{array}{c}10.25(2.73) \\
17.41(3.48) \\
5.2(1.39) \\
11.87(2.27) \\
\end{array}$ & $\begin{array}{l}10.79(2.16) \\
17.66(3.27) \\
5.26(1.58) \\
10.36(2.16) \\
\end{array}$ & $\begin{array}{l}\text { n.s. } \\
\text { n.s. } \\
\text { n.s. } \\
3.414 * * *\end{array}$ \\
\hline $\begin{array}{l}\text { Motivos } 3 \\
\text { 13. Motivos extrínsecos }(9+10+11) \\
\text { 14. Motivos intrínsecos }(12)\end{array}$ & $\begin{array}{l}32.86(5.67) \\
11.87(2.27)\end{array}$ & $\begin{array}{l}33.71(4.96) \\
10.36(2.16)\end{array}$ & $\begin{array}{c}\text { n.s. } \\
3.414^{* * * *}\end{array}$ \\
\hline Flow experimentado en el deporte y ejercicio físico & $43.33(6.01)$ & $40.81(6.39)$ & $2.043 *$ \\
\hline
\end{tabular}

$* p<0.05 ; * *<<0.01 ; * * *<0.001 ;$ n.s. $=$ diferencia no significativa

Fuente: elaboración propia. 
gorizaron los motivos en intrínsecos y extrínsecos, también se encontró que la diversión como motivo intrínseco era significativamente superior en el caso de los hombres.

Con respecto a los estados de flujo experimentados durante la realización de actividades físicodeportivas extraescolares, se encontraron diferencias significativas, siendo los hombres los que experimentan un mayor nivel de flujo.

\section{Relaciones entre práctica semanal de actividad físico-deportiva extraescolar, el flujo experimentado con la misma y los diferentes motivos}

En la Tabla 2 se muestran las correlaciones entre todas las variables del estudio, así como las asociaciones entre el flujo y los motivos extrínsecos e intrínsecos, y entre estos entre sí. Los minutos semanales de práctica físico-deportiva extraescolar se asociaron positivamente tanto con el flujo experimentado como con los motivos intrínsecos para la práctica, y esto tanto en mujeres como en hombres.

\section{Poder predictivo del flujo, los motivos intrínsecos y los motivos extrínsecos sobre la práctica semanal de actividad físico-deportiva extraescolar}

En la Tabla 3 se puede observar el resumen del análisis de regresión por pasos sucesivos para las variables correlacionadas con el volumen semanal de actividad físico-deportiva extraescolar. Para el grupo de hombres, el flujo ha sido el único factor
TABLA 2

Correlaciones de Pearson entre el tiempo de práctica semanal de actividad físico-deportiva extraescolar, el flujo experimentado y los motivos extrínsecos e intrínsecos de práctica

\begin{tabular}{|c|c|c|c|c|}
\hline & 1 & 2 & 3 & 4 \\
\hline & \multicolumn{4}{|c|}{ Hombres $(N=54)$} \\
\hline $\begin{array}{l}\text { 1. Minutos semanales de } \\
\text { práctica }\end{array}$ & \multicolumn{4}{|c|}{1} \\
\hline 2. Flujo experimentado & $0.44 * *$ & 1 & & \\
\hline 3. Motivos extrínsecos & 0.19 & $0.44 * *$ & 1 & \\
\hline \multirow[t]{2}{*}{ 4. Motivos intrínsecos } & $0.43 * *$ & $0.56 * *$ & $0.33 *$ & 1 \\
\hline & \multicolumn{4}{|c|}{ Mujeres $(N=47)$} \\
\hline $\begin{array}{l}\text { 1. Minutos semanales de } \\
\text { práctica }\end{array}$ & 1 & & & \\
\hline 2. Flujo experimentado & $0.33 *$ & 1 & & \\
\hline 3. Motivos extrínsecos & -0.05 & 0.03 & 1 & \\
\hline 4. Motivos intrínsecos & $0.36 *$ & 0.15 & 0.23 & 1 \\
\hline
\end{tabular}

$* p<0.05 ; * p<0.01$.

Fuente: elaboración propia.

que predice la práctica de actividad físico-deportiva, explicando un $19 \%$ de la varianza. También se introdujeron los motivos intrínsecos, pero estos no se mostraron como predictores significativos.

En el grupo de mujeres, la práctica de actividad físico-deportiva extraescolar fue predicha por los motivos intrínsecos y por la experimentación de estados de flujo. Ambos factores explicaron un $21 \%$ de la práctica de actividad física entre las mujeres.

\section{Discusión y conclusiones}

En la presente investigación se pretendió conocer el nivel de realización semanal de una actividad

TABLA 3

Resumen de análisis de regresión por pasos sucesivos para las variables que predicen la práctica de actividad físico-deportiva

\begin{tabular}{lccc}
\hline & $\beta$ & $t$ & $P$ \\
\hline ACTIVIDAD FÍSICO-DEPORTIVA EN HOMBRES & & & \\
$\left(R^{2}=0.19 ; F(1,52)=12.229 ; p=0.001\right)$ & & & \\
\hline Flujo experimentado durante la práctica físico-deportiva & 0.44 & 3.673 & 0.001 \\
\hline $\begin{array}{l}\text { ACTIVIDAD FÍSICO-DEPORTIVA EN MUJERES } \\
\left(R^{2}=0.21 ; F(2,44)=5.741 ; p=0.01\right)\end{array}$ & & & \\
\hline Motivos intrínsecos & & & \\
\hline Flujo experimentado durante la práctica físico-deportiva & 0.32 & 3.506 & 0.001 \\
\hline
\end{tabular}

Fuente: elaboración propia. 
físico-deportiva extraescolar en una muestra de jóvenes, así como los motivos para realizarla y las experiencias de flujo asociadas a dicha práctica. Además, se estudió la capacidad predictiva sobre dicha práctica de los estados de flow y de los motivos por los cuales los participantes han informado que realizaban actividad físico-deportiva.

Se ha encontrado que el $60.8 \%$ de los jóvenes que participaron en la evaluación llevan a cabo una actividad físico-deportiva extraescolar regular. Este porcentaje, sin diferenciación de sexos, se encuentra entre el informado por Sánchez-Barrera et al. (1995) del 67 \% y por Jiménez et al. (2008) del $53 \%$, aunque estos dos estudios se realizaron con población universitaria.

Por otro lado, entre los practicantes de actividad físico-deportiva extraescolar existe una gran variabilidad en cuanto a la frecuencia semanal, minutos dedicados por sesión e historial de práctica continuada. En el presente estudio, se han obtenido diferencias entre mujeres y hombres en cuanto a la cantidad de práctica de actividad físico-deportiva. Estos resultados apoyan los obtenidos en otros estudios en los que se destaca la diferencia en la actividad físico-deportiva realizada por ambos sexos, siendo los niveles de realización menores en las mujeres (Chillón et al., 2002; Nigg, 2003; Pastor et al., 1999; Piéron, 2002; Tercedor \& Delgado, 1998).

Con respecto a los motivos para la realización de actividad físico-deportiva extraescolar, los resultados indican que la totalidad de los practicantes de actividad físico-deportiva regular coinciden en señalar la diversión como un motivo muy importante para realizar dicha actividad. Estos resultados coinciden con los de Bakker, Whiting y Van der Brug (1992), Moreno, Cervelló y Martínez (2007) o Weinberg et al. (2000), y discrepan ligeramente de los de Elmer (2006), quien señala que el motivo de diversión sería más importante a una edad más temprana, siendo competir el motivo más frecuentemente señalado en la adolescencia y primera juventud.

Además, los participantes de nuestro estudio señalan como otros motivos muy importantes la mejora de la salud, de la forma física y del estado de ánimo. Estos resultados están en la línea de los obtenidos por Jiménez et al. (2007) y Moreno et al. (2007). Los practicantes de actividad físico-deportiva extraescolar del presente estudio señalan como poco importantes los motivos de competir y controlar el peso e imagen. En cambio, en otros estudios son señalados como motivos importantes competir (Pavón, Moreno, Gutiérrez \& Sicilia, 2003) o mejorar la apariencia corporal (Tiggemann \& Williamson, 2002). En el presente estudio, los practicantes de alguna actividad físico-deportiva extraescolar atribuyen mayor peso a los motivos intrínsecos (placer que produce o divertimento) que a motivos que se pueden considerar extrínsecos, siguiendo la Teoría de la Autodeterminación (p. e., mejorar el estado de ánimo, reducir ansiedad, mejorar la salud, controlar el peso, etc.).

Por otro lado, nuestros resultados indican que las mujeres tienen los mismos motivos que los varones para realizar ejercicio físico o actividades deportivas, aunque han aparecido diferencias en cuanto a dos motivos concretos para practicar actividad físico-deportiva, que han sido más valorados por los varones: divertirse y gusto de competir. En general, a la hora de justificar la práctica físico-deportiva extraescolar, los hombres suelen tener presentes un número significativamente mayor de motivos intrínsecos que las mujeres. En estas suelen tener más presencia los motivos extrínsecos, aunque no aparecieron diferencias con respecto a los hombres en esta categoría de motivos. Además, las mujeres han informado experimentar significativamente menos estados de flujo que los hombres al realizar ejercicio o deporte.

De los hallazgos anteriores puede concluirse la superioridad del constructo de motivación autodeterminada (Deci \& Ryan, 2002) en la predicción del inicio y la adherencia a programas deportivos y de ejercicio físico en jóvenes, así como la utilidad de la aplicación de la teoría del flujo al campo de la práctica de la actividad física y deportiva como elemento de salud en este colectivo.

Una cuestión clave que preocupa a investigadores y profesionales de la Psicología (Psicología del Ejercicio Físico y el Deporte, Psicología de la Salud), así como de las Ciencias del Ejercicio Físico y el Deporte, es cómo conseguir que los individuos inicien y mantengan la conducta física activa como hábito 
saludable y actividad de ocio positiva. En términos motivacionales, los hallazgos de otras investigaciones (Csikszentmihalyi \& Nakamura, 1989; Deci \& Ryan, 1985; Diener, Horwitz \& Emmons, 1985), y también en cierto modo los resultados del presente estudio, vienen a confirmar que una incidencia excesiva de las recompensas extrínsecas puede hacer disminuir la motivación intrínseca por la práctica de la actividad física y deportiva. En el caso de las personas más jóvenes, las diversas actuaciones que promueven la práctica físico-deportiva por motivos de salud o por otros motivos extrínsecos probablemente no tengan el calado esperado y deban ser cambiadas por otras en donde se subraye la experiencia autotélica (placer, diversión, satisfacción) que supone dicha práctica, al tiempo que se incite a establecer retos progresivos y se desarrollen las consiguientes habilidades para conseguirlos.

Se ha propuesto que el flujo motiva a la gente a actuar, es como una forma de energía (Csikszentmihalyi, 1996; Csikszentmihalyi \& Nakamura, 1989). La experimentación de estados de flujo puede constituir uno de los indicadores de la complejidad y desarrollo de las personas y puede ser un medio de crecimiento personal (Csikszentmihalyi, 1996). Además, experimentar estados subjetivos positivos como este es una de las principales razones por las que las personas mantienen la conducta a medio y largo plazo. La explicación de por qué algunas personas realizan actividades consumidoras de tiempo, de esfuerzo y hasta peligrosas, mientras que otras no lo hacen en absoluto, encuentra un marco justificativo en el tipo de vivencias experimentadas por esas personas al realizar dichas actividades. Por esto, se debe ayudar a los jóvenes a experimentar flujo en las diferentes actividades que acometan, tanto escolares como extraescolares. Se puede experimentar flujo de muchas maneras y, como hemos visto, la actividad físico-deportiva es uno de los contextos en que se experimenta más flujo porque con ella se persiguen unas metas, la persona conoce su nivel de ejecución, implica desafío en equilibrio con habilidades específicas, requiere concentración en la tarea, sensación de control y otras características del flujo, particularmente diversión (experiencias autotélicas).
La experiencia de flujo, y en particular la experiencia autotélica, pueden derivarse de las propias características de la actividad practicada (por eso nos encontramos con actividades más propensas a proporcionar flujo, como la práctica físico-deportiva), pero también puede explicarse a partir de las características y habilidades individuales de las personas, lo que se denomina "personalidad autotélica” (Csikszentmihalyi, 1997; Nakamura \& Csikszentmihalyi, 2005). Parece que las personas con más inclinación autotélica son más propensas a experimentar flujo en cualquier actividad que acometan (Nakamura \& Csikszentmihalyi, 2005).

Se necesita más investigación sobre las características específicas de los individuos con personalidad autotélica, y concretamente de sus procesos atencionales, que parecen ser un aspecto clave diferenciador de los individuos autotélicos para adentrarse en el canal del flujo y permanecer en él.

Igualmente, es necesario ampliar el número de instrumentos de evaluación del flujo experimentado en diferentes actividades cotidianas, y especialmente en el campo de la actividad física y el deporte, así como adaptar y aplicar esos instrumentos a distintos segmentos poblacionales (niños, adolescentes, adultos, mayores, discapacitados, deportistas profesionales, deportistas amateurs, etc.).

Hay que señalar algunas limitaciones en el presente estudio. En primer lugar, respecto a la selección y tamaño de la muestra, se considera que este estudio debería realizarse en el futuro con una muestra más extensa y seleccionada de modo estratificado. Otra limitación puede ser común a otras investigaciones en las que se evalúen las experiencias subjetivas para las que los informes están circunscritos al campo semántico. Así pues, la única información disponible de los cuestionarios son las palabras que se refieren a experiencias y no las experiencias en sí mismas.

Como conclusiones que merecen ser destacadas en el presente estudio, se puede señalar que solo el $50 \%$ de las mujeres y el $75 \%$ de los hombres evaluados han informado llevar a cabo una actividad físico-deportiva extraescolar regular, existiendo una gran variabilidad en cuanto a frecuencia semanal, tiempo de la sesión y la historia de práctica conti- 
nuada. Entre los practicantes, es en los motivos de diversión y gusto por competir en los que se producen unas diferencias más acusadas entre hombres y mujeres, siendo aquellos los más argumentados por los hombres. En cambio, no existen diferencias entre ambos sexos en cuanto al valor asignado a otro tipo de motivos para realizar ejercicio (mejora de la salud, de la forma física, del estado de ánimo, de la imagen corporal y control del peso o interacción social).

Además, los hombres experimentan un mayor nivel de flujo que las mujeres al realizar la actividad físico-deportiva. Tanto en los hombres como en las mujeres, el flujo experimentado predice la práctica físico-deportiva. Además, en el grupo de mujeres, el divertimento y otros motivos intrínsecos han explicado también la práctica deportiva.

Por lo tanto, de cara a potenciar la realización de ejercicio físico y deporte de un modo regular y mantenido a largo plazo en el colectivo de adolescentes y jóvenes, será necesario contemplar el trabajo sobre variables poco consideradas hasta el momento tales como el flujo y el divertimento. En cambio, los programas basados exclusivamente en la mejora de la salud física o psicológica, así como la reducción de peso o mejora de la figura, no conseguirán por sí solos asegurar la adherencia e impedir el abandono, puesto que no se ajustan a las principales razones por las que los y las jóvenes deciden iniciar la conducta física activa y mantenerla a medio y largo plazo. A la luz de los resultados obtenidos, para diseñar actuaciones que hicieran más probable que los jóvenes que inician un programa escolar o extraescolar de actividades físico-deportivas se adhieran convenientemente a él y no lo abandonen, es fundamental incrementar las habilidades para fomentar estados de flujo. Una forma de hacerlo en el ámbito de las actividades físicodeportivas consiste en el planteamiento de retos de progresiva dificultad y acordes con las habilidades de la persona para conseguirlos, el planteamiento de objetivos con una retroalimentación clara para verificar su cumplimento, ayudar a la persona a incrementar su autoeficacia y sensación de control, el entrenamiento en focalización de la atención y mejora de la concentración en la tarea a realizar y la automatización de los segmentos conductuales requeridos por la tarea. Por otra parte, resulta necesario fomentar motivos de práctica relacionados con la diversión y el disfrute por encima de cualesquiera otros motivos que también pudieran estar relacionados con dicha práctica.

\section{Referencias}

Bakker, F. C., Whiting, H. T. A. \& Van der Brug, H. (1992). Psicología del deporte. Conceptos y aplicaciones. Madrid: Morata.

Biddle, S. J. H. \& Mutrie, N. (2008). Psychology of physical activity: Determinants, well-being and interventions. London: Routledge.

Capdevila, L. (2003). Autoinforme de motivos para la práctica del ejercicio físico. En T. Gutiérrez, R. M. Raich, D. Sánchez \& J. Deus (Coords.), Instrumentos de evaluación en psicología de la salud (pp. 76-79). Madrid: Alianza Editorial.

Capdevila, L., Niñerola, J. \& Pintanel, M. (2004). Motivación y actividad física: el Autoinforme de Motivos para la Práctica de Ejercicio Físico (AMPEF). Revista de Psicología del Deporte, 13, 55-74.

Chillón, P., Tercedor, P., Delgado, M. \& González-Gross, M. (2002). Actividad físico-deportiva en escolares adolescentes. Retos. Nuevas Tendencias en Educación Física, Deporte y Recreación, 1, 5-12.

Cruz, J. (2001). Factores motivacionales en el deporte infantil y asesoramiento psicológico a entrenadores y padres. En J. Cruz (Ed.), Psicología del deporte (pp. 245-268). Madrid: Síntesis.

Csikszentmihalyi, M. (1975). Beyond boredom and anxiety. San Francisco: Jossey-Bass.

Csikszentmihalyi, M. (1996). Fluir (Flow) (11.a ed.). Barcelona: Kairós.

Csikszentmihalyi, M. (1997). Finding flow: The psychology of engagement with every-day life. New York: Basic Books.

Csikszentmihalyi, M. \& Csikszentmihalyi, I. S. (1998). La medición del flujo en la vida cotidiana [Introducción]. En M. Csikszentmihalyi \& I. S. Csikszentmihalyi (Eds.), Experiencia óptima. Estudios psicológicos del flujo en la conciencia (pp. 93-98). Bilbao: Desclée De Brouwer.

Csikszentmihalyi, M. \& Nakamura, J. (1989). The dynamics of intrinsic motivation. En R. Ames \& C. 
Ames (Eds.), Handbook of motivation theory and research (pp. 45-71). New York: Academic Press.

Daley, A. J. \& Duda, J. L. (2006). Self-determination, stage of readiness to change for exercise, and frequency of physical activity in young people. European Journal of Sport Sciences, 6, 231-243.

Deci, E. L \& Ryan, R. M. (1985). Intrinsic motivation and self-determination in human behavior. New York: Plenum.

Deci, E. L. \& Ryan, R. M. (2002). Handbook of selfdetermination research. Rochester NY: University of Rochester Press.

Diener, E., Horwitz, J. \& Emmons, R. A. (1985). Happiness of the very wealthy. Social Indicators Research, 16, 263-270.

Dishman, R. K., Sallis, J. P. \& Orensein, D. (1985). The determinants of physical activities and exercise. Public Health Report, 100, 158-171.

Dishman, R. K. (1988). Exercise adherence: Its impact on public health. Champaign, IL: Human Kinetics.

Dishman, R. K. (1991). Increasing and maintaining exercise and physical activity. Behavior Therapy, 22, 345-378.

Elmer, A. (2006). Motivos de participación y satisfacción en la actividad física, el ejercicio físico y el deporte. Revista MHSalud, 3, 1-16.

García-Calvo, T., Jiménez, R., Santos-Rosa, F. J., Reina, R. \& Cervelló, E. (2008). Psychometric properties of the Spanish version of the Flow State Scale. Spanish Journal of Psychology, 11, 660-669.

Godoy-Izquierdo, D., Molina, S. \& Godoy, J. F. (manuscrito en proceso de elaboración). Initiating and adhering to exercise: Motives, emotions and flow states.

Hagger, M. S. \& Chatzisarantis, N. L. (2007). Intrinsic motivation and self determination in exercise and sport. Champaigne, IL: Human Kinetics.

Jackson, S. A. \& Csikszentmihalyi, M. (2002). Fluir en el deporte. Claves para las experiencias y actuaciones óptimas. Barcelona: Paidotribo.

Jackson, S. A. \& Eklund, R. C. (2002). Assessing flow in physical activity: The Flow State Scale-2 and Dispositional Flow Scale-2. Journal of Sport and Exercise Psychology, 24, 133-150.

Jiménez, M. G., Benbunan, B., Sánchez, D., Martínez, J. M., Alfaya, M. P. \& Pérez, M. J. (2007, mayo).
Variables relacionadas con los motivos para la adherencia al ejercicio físico. IV Simposium Mundial Actividad Física, Salud y Empresa, Universidad de Granada, Melilla, España.

Jiménez, M. G., Martínez, M. P., Miró, E. \& Sánchez, A. I. (2008). Bienestar psicológico y hábitos saludables: ¿Están asociados a la práctica del ejercicio físico? International Journal of Clinical and Health Psychology, 8, 185-202.

Markland, D. \& Hardy, L. (1993). The Exercise Motivations Inventory: Preliminary development and validity of a measure of individuals' reasons for participation in regular physical exercise. Personality \& Individual Differences, 15, 289-296.

Morales, P. (2007). Estadística aplicada a las ciencias sociales. La fiabilidad de los tests y escalas. Madrid: Universidad Pontificia Comillas.

Moreno, J. A., Cervelló, E. \& Martínez, A. (2007). Validación de la Escala de Medida de los Motivos para la Actividad Física-Revisada en españoles: diferencias por motivos de participación. Anales de Psicología, 23, 167-176.

Nakamura, J. \& Csikszentmihalyi, M. (2005). The concept of flow. En C. R. Snyder \& S. J. López (Eds.), Handbook of positive psychology (pp. 89-105). New York: Oxford University Press.

Nigg, C. R. (2003). Do sport participation motivations add to the ability of the transtheoretical model to explain adolescent exercise behaviour? International Journal of Sport Psychology, 34, 208-225.

Pastor, Y., Balaguer, I. \& García-Merita, M. (1999). Influencia del autoconcepto multidimensional y de la autoestima sobre la práctica deportiva en la adolescencia media: análisis diferenciales por género. En G. Nieto \& E. J. Garés (Eds.), Psicología de la actividad física y el deporte (Vol. 1, pp. 182-189). Murcia: Sociedad Murciana de Psicología de la Actividad Física y el Deporte.

Pavón, A. I., Moreno, J. A., Gutiérrez, M. \& Sicilia, A. (2003). Intereses y motivaciones de los universitarios: diferencias en función del nivel de práctica. Cuadernos de Psicología del Deporte, 3, 33-43.

Piéron, M. (2002). Estilo de vida, práctica de actividades físicas y deportivas, calidad de vida. Recuperado el 4 de septiembre de 2007, de http://www.sportsalut. com.ar/articulos/educacion_fisica/2.pdf 
Ryan, R. M. \& Deci, E. L. (2000). Self-determination theory and the facilitation of intrinsic motivation, social development and well-being. American Psychologist, 55, 68-78.

Sánchez-Barrera, M., Pérez, M. \& Godoy, J. (1995). Patrones de actividad física en una muestra española. Revista de Psicología del Deporte, 7, 51-71.

Seligman, M. E. P. \& Csikszentmihalyi, M. (2000). Positive psychology: An introduction. American Psychologist, 55, 5-14.

Sicilia, A., Águila, C., Muyor, J. M., Orta, A. \& Moreno, J. A. (2009). Perfiles motivacionales de los usuarios en centros deportivos municipales. Anales de Psicología, 25, 160-168.

Tercedor, P. \& Delgado, M. (1998). El sedentarismo en los escolares: estudio en población de 5o curso de Educación Primaria. En A. García, F. Ruiz \& A. J. Casimiro (Eds.), Actas del II Congreso Internacional sobre la Enseñanza de la Educación Física y el Deporte Escolar (pp. 277-280). Málaga: Instituto Andaluz del Deporte.
Tiggemann, M. \& Williamson, S. (2002). The effect of exercise on body satisfaction and self-esteem as a function of gender and age. Sex Roles, 43, 119-127.

Varo, J. J., Martínez-González, M. A., de Irala-Estévez, J., Kearney, J., Gibney, M. \& Martínez, J. A. (2003). Distribution and determinants of sedentary lifestyles in the European Union. International Journal of Epidemiology, 32, 138-146.

Weinberg, R., Tenenbaum, G., McKenzie, A., Jackson, S., Anshel, M., Grove, R. \& Fogarty, G. (2000). Motivation for youth participation in sport and physical activity: Relationships to culture, selfreported activity levels and gender. International Journal of Sport Psychology, 31, 321-346.

Wilson, P. M., Rodgers, W. M., Fraser, S. N. \& Murray, T. C. (2004). Relationships between exercise regulations and motivational consequences in university students. Research Quarterly for Exercise and Sport, 75, 81-91. 